\title{
A Benchmark TEAM Problem for Multi-Objective Pareto Optimization of Electromagnetic Devices
}

\author{
Paolo Di Barba ${ }^{1}$, Maria Evelina Mognaschi ${ }^{1}$, David A. Lowther ${ }^{2}$, Fellow, IEEE, \\ and Jan K. Sykulski ${ }^{3}$, Fellow, IEEE \\ ${ }^{1}$ Department of Electrical, Computer and Biomedical Eng, University of Pavia, 27100 Pavia, Italy \\ ${ }^{2}$ Department of Electrical and Computer Engineering, McGill University, Montreal, QC H3A OE9, Canada \\ ${ }^{3}$ Electronics and Computer Science, University of Southampton, Southampton SO17 1BJ, U.K.
}

This paper proposes a new benchmark for multi-objective optimization. A solution is furnished which has enabled an extensive search and reliable estimation of the shape of the Pareto front. Field uniformity and sensitivity are considered in the context of robust design. It is argued that the benchmark will provide a challenging target for new algorithms, especially those involving numerical modeling using finite-element codes where the number of objective function calls needs to be minimized for practical design processes.

Index Terms—Design optimization, multi-objective optimization, Pareto front, sensitivity analysis.

\section{INTRODUCTION}

$\mathbf{T}$ EAM problems are well established and available from the website of the International Compumag Society [1]. In the area of optimization of electromagnetic devices there are two particular benchmarks, known as No 22 SMES Optimization Benchmark and No 25 Optimization of Die Press Model. Both have been used extensively for testing new single-objective optimization algorithms. For some time there has been a need to create a benchmark which could ultimately be used for multi-objective problems, [2] and [3], with particular emphasis on Pareto optimization. In fact, a multi-criteria design is usually needed in many problems of real-life engineering: from classical applications such as electrical machines [4] and [5] to the newest devices in the micro electro-mechanical systems or nano electro-mechanical systems area [6], the approach in terms of multi-objective optimization is more and more used. Moreover, according to the rationale of the no-free lunch theorem, new algorithms need to be tested on specific issues [7]-[9].

In this paper, we put forward a simple-but often important in practical applications-model for assessing the quality of a magnetic field produced by a distributed winding. The field uniformity is usually of prime interest but sensitivity is also an important issue, especially in the context of robust design. The available design space has been extensively searched to yield a reliable non-dominated solution for further consideration in a Pareto sense. Optimization algorithms may therefore be tested against the proposed benchmark. Other objectives may also be defined, for example to reduce the power loss and make a design more efficient. Finally, three objectives may be attempted simultaneously. This paper is organized as follows. In Section II, the field model of the electromagnet is presented. In Section III, the objective functions ruling field uniformity, design sensitivity, and power loss are defined in

Manuscript received June 27, 2017; revised August 4, 2017; accepted August 21, 2017. Corresponding author: M. E. Mognaschi (e-mail: eve.mognaschi@unipv.it).

Color versions of one or more of the figures in this paper are available online at http://ieeexplore.iee.org.

Digital Object Identifier 10.1109/TMAG.2017.2750901 terms of design variables; eventually, Section IV is devoted to optimization results.

\section{Electromagnet Optimal Design: MODEL SPECIFICATION}

Suitably arranged current-carrying coils can be utilized to synthesize a magnetic field with a desired distribution. In biomedical engineering, for instance, there are many relevant applications: a uniform magnetic field is the background of nuclear magnetic resonance spectroscopy, while a linear profile of the field is a prerequisite for magnetic resonance imaging. Moreover, in magneto-fluid hyperthermia (MFH) the field uniformity helps to achieve a uniform distribution of heat generated in the nano-particle fluid previously injected into the target region, e.g., a tumor mass under treatment [10]. Thus, the idea behind this benchmark problem has been inspired by important practical applications.

Consider an air-cored multi-turn winding. A non-trivial inverse problem is the synthesis of the magnetic field along the solenoid axis. This problem can be formulated as follows: given the current density $J$, find the distribution of turn radii $r(z),-d \leq z \leq d$ that yields the prescribed flux density, $B_{0}(z)$, in a 1 -D sub-region $-c \leq z \leq c$ along the solenoid axis. In principle, for the flux density due to a multi-turn winding with an internal radius $r_{i}$, an external radius $r_{s}$, and carrying a current $J d z$, the following expression for the flux density at point $z$ holds:

$$
B(z)=\frac{\mu_{0}}{2} \int_{-d}^{d} \int_{r_{i}}^{r_{s}} \frac{J r^{2}(\xi) d r d \xi}{\sqrt{\left[r^{2}(\xi)+(z-\xi)^{2}\right]^{3}}} .
$$

If $B(z)=B_{0}(z),-c \leq z \leq c$, is the prescribed function and $r(\xi),-d \leq \xi \leq d$, is the unknown function, then (1) implies that the field distribution which is required in a region inside the winding can be synthesized by imposing a suitable distribution of the turn radii. From (1), the following remarks can be put forward.

1) Analysis Problem: given the current distribution $J$ and the $\mathrm{r}$ distribution of radii, find flux density $\mathrm{B}$ in the absence of ferromagnetic cores. Thus, this requires the solution of a linear problem, the computational cost of 


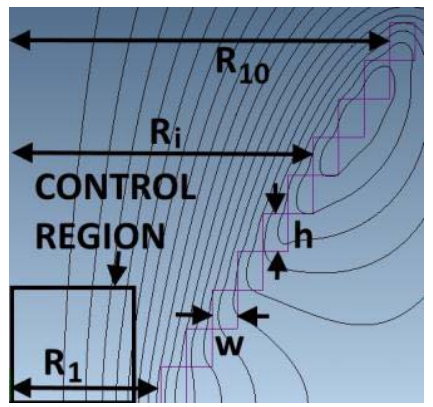

Fig. 1. Geometry and magnetic flux lines: the design variables and the controlled region are shown.

which is low. This condition is a practical prerequisite to solve multi-objective optimization problems: in fact, in spite of their popularity, the performance of evolutionary algorithms deteriorates as the number of objectives increases [11].

2) Current-Oriented Synthesis Problem: the flux density $\mathrm{B}$ is prescribed, the current distribution $\mathrm{J}$ is unknown, and the $r$ distribution of radii is assigned. The problem results in a Fredholm's integral equation of the first kind which can be solved using standard techniques of regularization [12]-[14]. The computational cost is high due to the iterative solution.

3) Radius-Oriented Synthesis Problem: the flux density $\mathrm{B}$ is prescribed, the current distribution $\mathrm{J}$ is assigned, and the $r$ distribution of radii is unknown. Due to the non-linear relationship between $B$ and $r$, the problem does not lead to a Fredholm's integral equation of the first kind and, therefore, it is much more complicated and computationally expensive. Moreover, the problem is possibly ill posed due to the possible existence of multiple solutions.

Here, the radius-oriented synthesis problem, assuming a 2-D controlled region for the prescribed flux density, is considered. To focus the attention, a small-size solenoid, as used for in vitro experiments of $\mathrm{MFH}$, is considered here, with the width of each turn $w=1 \mathrm{~mm}$ and the height $h=1.5 \mathrm{~mm}$.

The winding is composed of $n_{t}=20$ series-connected turns. Thus, assuming a symmetric distribution, ten unknown radii (design variables) are to be identified. A 2-D controlled region is considered: the flux density $B_{0}(r, z)=\left(0, B_{p}\right)$ with $B_{p}=2 \mathrm{mT}$ is prescribed at $n_{p}=66$ sample points, evenly spaced in a $5 \times 5 \mathrm{~mm}$ squared region; due to a symmetric distribution only half of the model is needed. The geometric model of the winding is shown in Fig. 1.

The analysis problem-i.e., given $J$ and $r$ distributions find the flux density field $B$-was solved using a finite-element $(r, z)$ axisymmetric model [15].

Because a stable solution is needed, the mesh has been tuned a priori by considering a family of meshes exhibiting different element densities (Fig. 2). The maximum element size chosen for the controlled region is equal to $0.1 \mathrm{~mm}$. A typical mesh is composed of 100000 second-order elements.

\section{UnIFORMity Versus SENSITIVITY}

The uniformity of the field is usually of prime interest, but sensitivity cannot be overlooked [16]. The issue of design sensitivity in the multi-objective scenario is relatively recent: it was considered e.g., in [17] and also in [18] and [19],

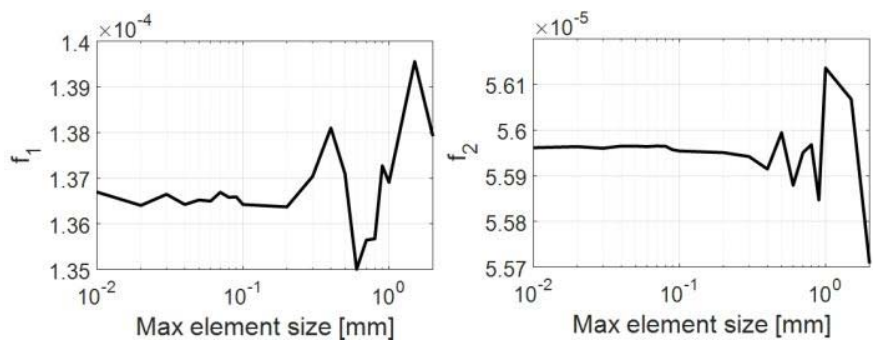

Fig. 2. Finite-element mesh tuning in terms of maximum element size.

where surrogate models were exploited. Therefore, we also propose that an additional design criterion should be introduced, namely, the sensitivity of the electromagnet to small errors in placing or shaping the turns. The goal then becomes to reduce the sensitivity as much as possible, without upsetting the requirement of a particular field distribution within a certain tolerance, thus making the design more robust. Eventually, power loss is relevant too to an efficient design; therefore, we propose an alternative formulation of the synthesis problem, in which a power-loss related objective function is considered.

Accordingly, the design problem was reformulated in terms of two bi-objective optimization problems, as follows.

Problem 1: find the family of $r$-distributions that minimize the discrepancy—or field residual-between the prescribed $B_{0}$ and the actual induction field $B$

$$
f_{1}(r)=\sup _{q=1, n_{p}}\left|B\left(r\left(\xi_{\ell}\right), z_{q}\right)-B_{0}\left(r_{q}, z_{q}\right)\right|, \quad \ell=1, n_{t}
$$

and simultaneously minimize the following sensitivity function

$$
\begin{aligned}
f_{2}(r)= & \sup _{\ell=1, n_{t}}\left[\left\|B^{+}-B\left(r\left(\xi_{\ell}\right), z_{q}\right)\right\|\right. \\
& \left.+\left\|B\left(r\left(\xi_{\ell}\right), z_{q}\right)-B^{-}\right\|\right] \\
q= & 1, n_{p}
\end{aligned}
$$

where $B^{+}$and $B^{-}$are the flux density values computed after an expansion, or a contraction, of all radii with respect to the unperturbed configuration, respectively, that is

$$
\begin{aligned}
& B^{+}=B\left(r\left(\xi_{\ell}+\Delta \xi\right), z_{q}\right) \\
& B^{-}=B\left(r\left(\xi_{\ell}-\Delta \xi\right), z_{q}\right), \quad \ell=1, n_{t}, q=1, n_{p}
\end{aligned}
$$

where the amount of expansion/contraction is constant and equal to $\Delta \xi=0.5 \mathrm{~mm}$. In (2) a constant field of value $B_{p}$ within the controlled region $[-5,5] \mathrm{mm} \times[-5,5] \mathrm{mm}$ inside the winding is considered.

Problem 2: find the family of $r$-distributions that minimize the discrepancy $f_{1}(r)$-or field residual-between the prescribed $B_{p}$ and the actual induction field B and simultaneously minimize the power-loss related function

$$
f_{3}(r)=\sum_{\ell=1}^{n_{t}} r\left(\xi_{\ell}\right)
$$

Note that (5) is proportional to the ohmic resistance of the winding, which is supplied at a constant DC current. For both Problems 1 and 2, the lower and upper bounds of the design variables, defining the feasible region, are set to: 5 and $50 \mathrm{~mm}$, respectively. 
TABLE I

Starting Solutions For Single-ObJective Optimizations

\begin{tabular}{|c|c|c|}
\hline Case & Design variables $[\mathrm{mm}]$ & $\begin{array}{c}\text { Objective functions } \\
{\left[\mathrm{f}_{1}, \mathrm{f}_{2}, \mathrm{f}_{3}\right]}\end{array}$ \\
\hline $\begin{array}{l}\text { Constant } \\
\text { Linear } \\
\end{array}$ & $\begin{array}{c}{\left[\begin{array}{llllllllll}10 & 10 & 10 & 10 & 10 & 10 & 10 & 10 & 10 & 10\end{array}\right]} \\
{\left[\begin{array}{llllllllll}6 & 7 & 8 & 9 & 10 & 11 & 12 & 13 & 14 & 15\end{array}\right]} \\
\end{array}$ & $\begin{array}{l}{\left[1.3610^{-4}, 5.5910^{-5}, 100\right]} \\
{\left[8.1810^{-4}, 3.0110^{-4}, 105\right]} \\
\end{array}$ \\
\hline
\end{tabular}

TABLE II

RESUlts of Single-ObJective Optimizations

\begin{tabular}{|c|c|c|c|}
\hline $\begin{array}{l}\text { Starting } \\
\text { point/Case }\end{array}$ & $\begin{array}{l}\text { Optimized } \\
\text { function }\end{array}$ & Design variables [mm] & $\begin{array}{c}\text { Objective functions } \\
{\left[\mathrm{f}_{1}, \mathrm{f}_{2}, \mathrm{f}_{3}\right]}\end{array}$ \\
\hline Constant/C & 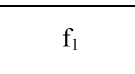 & 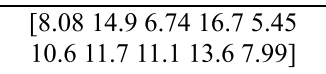 & $\begin{array}{l}{\left[3.1310^{-5}, 8.5410^{-5},\right.} \\
106.8]\end{array}$ \\
\hline Linear/C & $f_{1}$ & 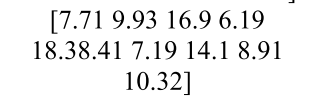 & $\begin{array}{l}{\left[7.2810^{-5}, 1.9110^{-4},\right.} \\
108]\end{array}$ \\
\hline Constant /D & $f_{1}$ & 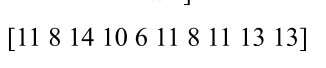 & $\begin{array}{l}{\left[3.1610^{-5}, 1.2110^{-5},\right.} \\
105]\end{array}$ \\
\hline Constant/C & $\mathrm{f}_{2}$ & $\begin{array}{l}{\left[\begin{array}{llllll}31.3 & 29.7 & 33.9 & 33.0 & 20.6 \\
16.3 & 12.4 & 5.53 & 13.2 & 5.54\end{array}\right]}\end{array}$ & $\begin{array}{l}{\left[9.4510^{-4}, 3.8710^{-6},\right.} \\
201.4]\end{array}$ \\
\hline Linear/C & $\mathrm{f}_{2}$ & $\begin{array}{lllll}{\left[\begin{array}{lllll}16.1 & 16.2 & 30.9 & 14.3 & 12.5 \\
11.5 & 25.1 & 5.50 & 44.3 & 6.96\end{array}\right]}\end{array}$ & $\begin{array}{l}{\left[5.7710^{-4}, 2.9810^{-5},\right.} \\
183.3]\end{array}$ \\
\hline Constant/D & $\mathrm{f}_{2}$ & 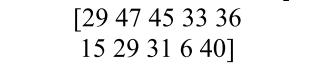 & $\begin{array}{l}{\left[1.0810^{-3}, 1.5210^{-5},\right.} \\
311]\end{array}$ \\
\hline $\begin{array}{l}\text { Known } \\
\text { exactly }\end{array}$ & $f_{3}$ & 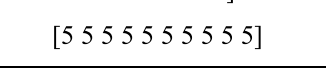 & $\begin{array}{l}{\left[2.0710^{-4}, 2.1210^{-3},\right.} \\
50]\end{array}$ \\
\hline
\end{tabular}

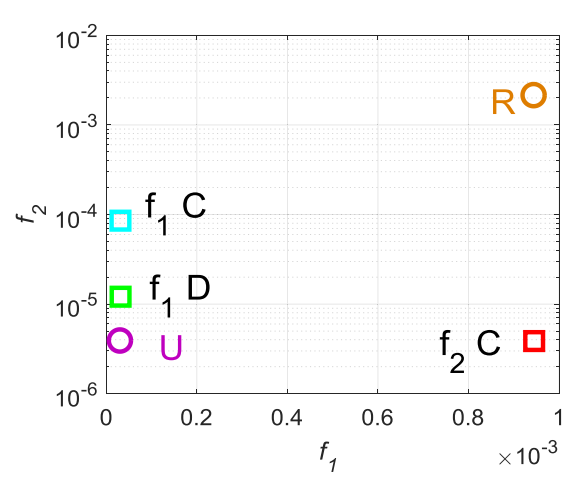

Fig. 3. Results of single-objective optimizations, plotted as $f_{2}$ versus $f_{1}$.

\section{REsults AND Discussion}

A study based on repeated single-objective optimizations has been performed first. Then, a bi-objective optimization problem was worked out for both Problem 1 and Problem 2.

\section{A. Single-Objective Optimization Results}

The single-objective problems are solved using a $(1+1)$ evolution strategy (EStra algorithm) [20]. Two cases are considered: the design variables can be either continuous (C) or discrete (D). The step value of the discrete design variables is equal to $1 \mathrm{~mm}$. The two starting solutions used for the continuous case are: the constant radii equal to $10 \mathrm{~mm}$ (named "constant case") and the radii varying from 6 to $15 \mathrm{~mm}$ (named "linear case"). The design variables and the objective functions for the constant and the linear cases are shown in Table I. The starting solution for the optimization with discrete variables is the constant case. The results of the single-objective optimization are shown in Table II and Figs. 3 and 4.

It could be noted that the solution of the minimization problem for function $f_{3}$ is known exactly. In fact, being the lower bound of the design variables equal to $5 \mathrm{~mm}$, this is also the exact solution that minimizes power loss in the winding.

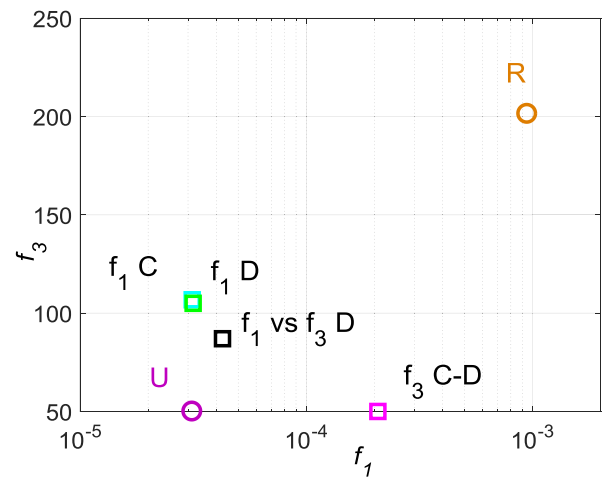

Fig. 4. Results of single-objective optimizations, plotted as $f_{3}$ versus $f_{1}$.

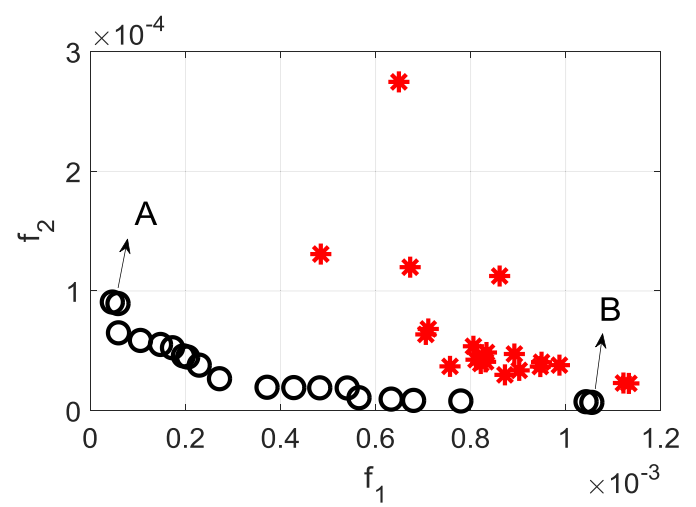

Fig. 5. Results of a bi-objective optimization (Problem 1): star-starting solutions; circle-final solutions.

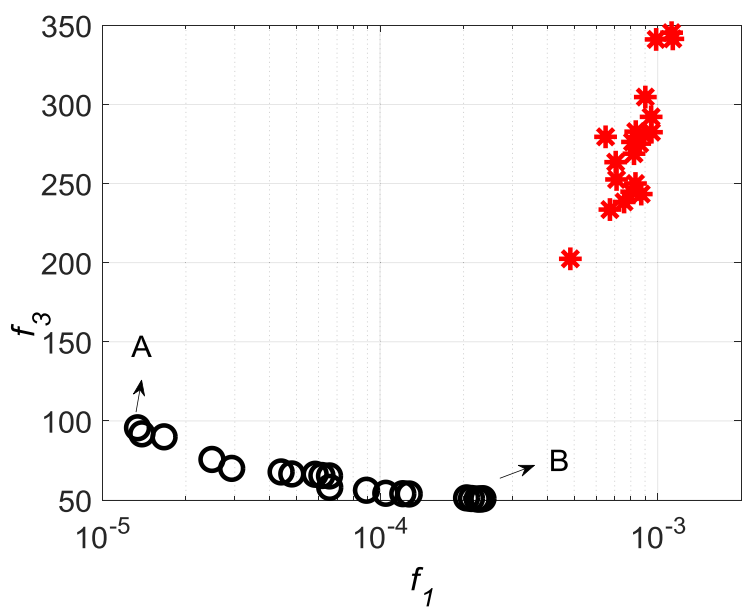

Fig. 6. Results of a bi-objective optimization (Problem 2): star-staring solutions; circle-final solutions.

In general, the solutions found with constant starting solution are moderately better than those found starting from the linear case. Moreover, the solutions obtained with continuous variables are slightly better than those with discrete variables. Hence, the solutions obtained in the constant case with continuous and discrete variables are plotted in the objective space $f_{2}$ versus $f_{1}$ (Fig. 3) and $f_{3}$ versus $f_{1}$ (Fig. 4). In particular, only non-dominated solutions are shown. The approximated utopia (U) and nadir (R) points are shown in each objective space; the relevant coordinates can be read in Table I. The existence of $\mathrm{U}$ and $\mathrm{R}$ points proves the conflicts in the given optimization problems.

For the sake of a comparison, in Fig. 4 a single-solution of the multi-objective $\left(f_{1}, f_{3}\right)$ problem is also shown: it was 


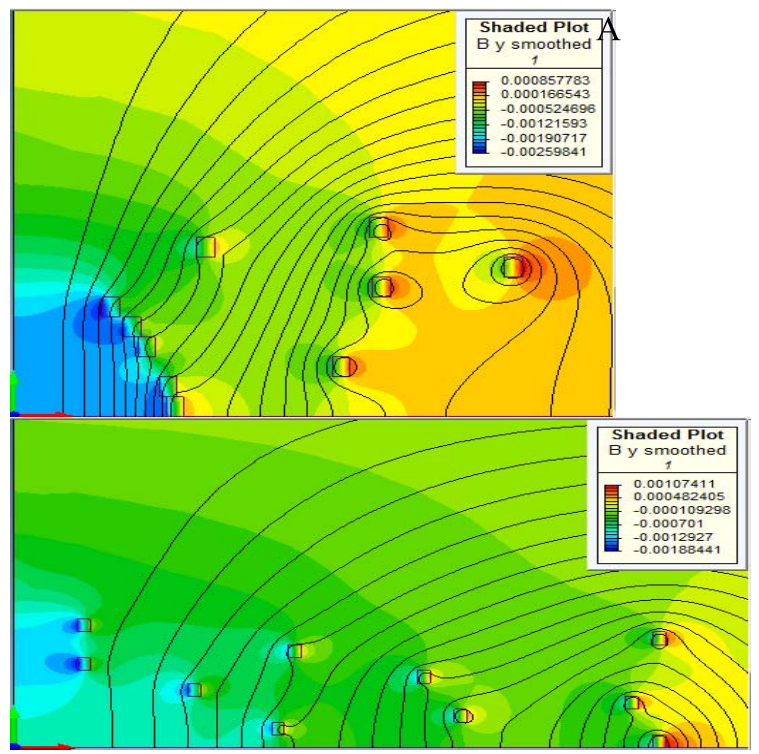

Fig. 7. Field map of magnetic induction for solutions A and B of Fig. 5.

obtained by means of a modified $(1+1)$ EStra algorithm [21], in which an offspring solution is accepted if and only if it Pareto-dominates the parent solution.

\section{B. Bi-Objective Optimization Results}

NSGA-II algorithm [22] was subsequently used. The number of iterations is 80 for Problem 1 and 110 for Problem 2; the population size is 20 while the parameters $m и$ and mum, which rule crossover and mutation probability, respectively, are set to 0.2 . The starting points are randomly generated in the feasible region. The results are shown in Figs. 5 and 6.

End-point solutions A and B of Fig. 5 are shown in Fig. 7.

\section{CONCLUSION}

A testing electromagnetic analysis methods benchmark problem has been proposed, and a set of feasible solutions derived, to establish a Pareto front. The uniformity of the field is usually of prime interest, but sensitivity is also an important issue, especially in the context of robust design, while power loss is relevant to an efficient design. Accordingly, three objective functions have been specified which give rise to a pair of 2-D objective spaces, eventually exhibiting a particular shape of the Pareto front. The design space has been thoroughly searched to provide a definite location of the non-dominated solution. Various optimization algorithms may therefore be tested against the proposed benchmark.

Generally, the proposed benchmark shows that even in the case of quite simple geometries-and straightforward analysis tasks - complicated inverse problems may arise. Solutions of the proposed benchmark with different numerical methods and results obtained by different authors are welcome, in order to set up a reference collection of data in the area of Pareto optimization in magnetostatics.

\section{REFERENCES}

[1] International Compumag Society. Accessed: Sep. 21, 2017. [Online]. Available: http://www.compumag.org/jsite/team.html

[2] P. Di Barba et al., "A benchmark problem of induction heating analysis," Int. J. Appl. Electromagn. Mech., vol. 53, no. S1, pp. 139-149, 2017.

[3] P. Di Barba et al., "Multiphysics field analysis and multiobjective design optimization: A benchmark problem," Inverse Problems Sci. Eng., vol. 22, no. 7, pp. 1214-1225, 2014.

[4] P. Di Barba, M. E. Mognaschi, R. Palka, P. Paplicki, and S. Szkolny, "Design optimization of a permanent-magnet excited synchronous machine for electrical automobiles," Int. J. Appl. Electromagn. Mech., vol. 39, nos. 1-4, pp. 889-895, 2012.

[5] M. H. Mohammadi, T. Rahman, R. Silva, M. Li, and D. A. Lowther, "A computationally efficient algorithm for rotor design optimization of synchronous reluctance machines," IEEE Trans. Magn., vol. 52, no. 3, Mar. 2016, Art. no. 8200804.

[6] P. Di Barba, F. Dughiero, M. E. Mognaschi, A. Savini, and S. Wiak, "Biogeography-inspired multiobjective optimization and MEMS design," IEEE Trans. Magn., vol. 52, no. 3, Mar. 2016, Art. no. 7201504.

[7] P. Di Barba, B. Liu, M. E. Mognaschi, P. Venini, and S. Wiak, "Multiphysics field analysis and evolutionary optimization: Design of an electro-thermo-elastic microactuator," Int. J. Appl. Electromagn. Mech., vol. 54, no. 3, pp. 433-448, 2017.

[8] P. Di Barba, "Multi-objective wind-driven optimisation and magnet design," Electron. Lett., vol. 52, no. 14, pp. 1216-1218, 2016.

[9] P. Di Barba, M. E. Mognaschi, A. Savini, and S. Wiak, "Island biogeography as a paradigm for MEMS optimal design," Int. J. Appl. Electromagn. Mech., vol. 51, no. 1, pp. S97-S105, 2016.

[10] R. Bertani et al., "Optimal inductor design for nanofluid heating characterisation," Eng. Comput., vol. 32, no. 7, pp. 1870-1892, 2015.

[11] R. Silva, A. Salimi, M. Li, A. R. R. Freitas, F. G. Guimarães, and D. A. Lowther, "Visualization and analysis of tradeoffs in manyobjective optimization: A case study on the interior permanent magnet motor design," IEEE Trans. Magn., vol. 52, no. 3, Mar. 2016, Art. no. 8102404 .

[12] R. Sikora, J. Purczynśki, and K. Adamiak, "The magnetic field synthesis on a cylinder solenoid axis by means of Tichonov's regularization method," Arch. Elektrotech., vol. 60, no. 2, pp. 83-86, 1978.

[13] P. Neittaanmaki, M. Rudnicki, and A. Savini, Inverse Problems and Optimal Design in Electricity and Magnetis. Oxford, U.K.: Clarendon, 1996.

[14] P. Di Barba, Multiobjective Shape Design in Electricity and Magnetism. Berlin, Germany: Springer, 2010.

[15] Infolytica Corporation. Accessed: Sep. 21, 2017. [Online]. Available: http://www.infolytica.com

[16] A. Formisano, P. Di Barba, R. Martone, M. Repetto, A. Salvini, and A. Savini, "A brief survey of robust optimization," Int. J. Appl. Electromagn. Mech., to be published.

[17] A. Salimi and D. A. Lowther, "On the role of robustness in multiobjective robust optimization: Application to an IPM motor design problem," IEEE Trans. Magn., vol. 52, no. 3, Mar. 2016, Art. no. 8102304.

[18] M. H. S. Mendes, G. L. Soares, J. L. Coulomb, and J. A. Vasconcelos, "A surrogate genetic programming based model to facilitate robust multiobjective optimization: A case study in magnetostatics," IEEE Trans. Magn., vol. 49, no. 5, pp. 2065-2068, May 2013.

[19] B. Xia, Z. Ren, and C.-S. Koh, "Utilizing Kriging surrogate models for multi-objective robust optimization of electromagnetic devices," IEEE Trans. Magn., vol. 50, no. 2, Feb. 2014, Art. no. 7017104.

[20] P. Di Barba, A. Savini, and S. Wiak, Field Models in Electricity and Magnetism. Berlin, Germany: Springer, 2008.

[21] P. Di Barba and M. E. Mognaschi, "Recent experiences of multiobjective optimisation in electromagnetics: A comparison of methods," Int. J. Comput. Math. Elect. Electron. Eng., vol. 24, no. 3, pp. 921-930, 2005.

[22] MathWorks. Accessed: Sep. 21, 2017. [Online]. Available: http://www.mathworks.com 